\title{
A SCAFFOLDING FRAMEWORK FOR SOCIAL ANNOTATION IN ONLINE CLASSES
}

\author{
Xinran Zhu, Hong Shui, Bodong Chen
}

University of Minnesota

\section{INTRODUCTION}

Web annotation is a genre of information technology that allows a user to annotate information in a shared document and anchor a discussion to the annotated information. When it is used socially in online classes, web annotation can support social reading, group sensemaking, knowledge construction, and community building (Chen, 2019; Kalir et al., 2020, Zhu et.al, 2020).

To support several University of Minnesota instructors' pivot to online teaching, we conducted a study that was focused on engaging undergraduate students in reading and discussing course materials in online classes. Following a co-design approach, we worked closely with instructors to design a generic scaffolding framework for social annotation activities and supported them to implement the framework, with course-specific customizations, in their classes. All participating courses are piloting a web annotation tool named Hypothes. is ${ }^{1}$ that is integrated in Canvas and allows students to read and annotate socially. This document describes the scaffolding framework, an important outcome of the study, which can be applied in various online/hybrid class settings.

\section{THE SCAFFOLDING FRAMEWORK}

The generic scaffolding framework has two main scaffolding strategies-dynamic grouping and participation roles-that can be further customized in response to each course (see Fig. 1).

- The Dynamic Grouping Strategy assigns different articles (e.g., primary and secondary materials) to different groups. Each group summarizes key points from annotations in the assigned articles and shares the summary in a class discussion forum on Canvas for comparing and connecting perspectives.

- The Participation Roles Strategy we designed has three roles (Wise et al., 2012): a facilitator responsible for stimulating conversations by finding connections, seeking clarifications, and pursuing consistent tags; a synthesizer who synthesizes the initial ideas, highlights agreement/disagreement, and suggests directions of further discussions in the middle of the week; and a summarizer who summarizes group conversations at the end of the week for the whole class. 


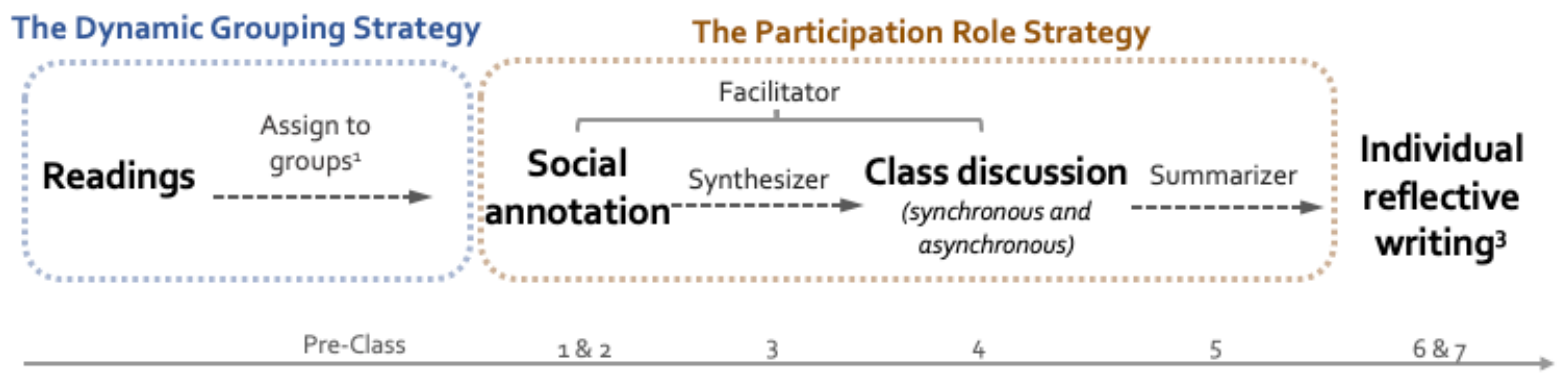

Timeframe - day in a week ${ }^{2}$

${ }^{2}$ Instructor may decide how to assign groups and readings.

${ }^{2}$ Example timeframe for the strategies, e.g., synthesizer completes the task on the third day. Instructors may adjust the timeframe accordingly.

3 Instructors may adjust this accordingly.

Figure 1. Design of Social Annotation Activities.

When it is enacted in a course, the generic framework can be further customized based on unique course objectives, features, and constraints. As illustrated in Fig. 1, instructors decide how to flexibly use the dynamic grouping and participation roles based on the class features. For the Dynamic Grouping Strategy, we suggest to divide the class into groups if the class size is large. The instructors may decide if to assign same readings to all groups or assign different readings to each group. For the Participation Role Strategy, each week, the instructor assigns the roles to different students within each group. The students annotate the course readings and interact with each other by replying to the annotations. The annotation activity is promoted by the facilitator throughout the week. In the Hypothes.is discussion, students negotiate the meaning of the key terms from different perspectives for a shared and deep understanding. The synthesizer collects students' diverse perspectives to reflect their initial thoughts. In the class Zoom meeting or Canvas discussion forum, students discuss the annotations for a further collective reflection to unpack puzzlement and connect/compare various perspectives. After the summarizer summarizes each week's activities on Canvas discussion forum, each student writes their individual reflective writing to connect social annotation with individual reflection.

\section{IMPLICATIONS}

To foster collaborative learning in courses supported by social annotation, the Dynamic Grouping Strategy keeps the amount of conversation on Hypothes. is manageable and allows students to "jigsaw" the knowledge contributed by other groups. The Participation Roles Strategy improves the group-level discussions and serves as pipelines to connect group annotations, class-level collaboration and individual reflections.

Grounded in the Computer-Supported Collaborative Learning (CSCL) literature and established based on a strong rapport between instructors and researchers, the framework has implications on both practice and research. On the one hand, it provides a customizable workflow for instructors to integrate social annotations activities into their teaching practice. On the other hand, it provides implications for researchers on collaborating with instructors and distilling design patterns that can be applied to a broader context. 
Zhu, X., Shui, H., \& Chen, B. (2020, December 23). A Scaffolding Framework for Social Annotation in Online Classes. https://doi.org/10.35542/osf.io/zk4vj

\section{ACKNOWLEDGMENTS}

We would like to thank our collaborators from the College of Liberal Arts at the University of Minnesota that have provided us with great support and insightful ideas through the design meetings. We greatly appreciate Dr. Cindy Garcia and Jason Noer, instructor and TA of Dance History 1; Dr. Malinda Lindquist, instructor of The Sixties: History \& Memory; and Dr. Ronald Green, instructor of Introduction to Rhetorical Theory, who have been co-designing social annotation activities and sharing experiences with us throughout the semester. We also would like to thank Shana Crosson, Academic Technologist, who has been passionate and provided full support to this project.

\section{REFERENCES}

Chen, B. (2019). Designing for networked collaborative discourse: An UnLMS approach. TechTrends, 63(2), 194-201.

Kalir, J. R., Morales, E., Fleerackers, A., \& Alperin, J. P. (2020). When I saw my peers annotating: Student perceptions of social annotation for learning in multiple courses. Information and Learning Sciences, doi: 10.1108/ILS12-2019-0128.

Wise, A. F., Saghafian, M., \& Padmanabhan, P. (2012). Towards more precise design guidance: Specifying and testing the functions of assigned student roles in online discussions. Educational Technology Research and Development, 6o(1), 55-82.https://doi.org/10.1007/s11423-011-9212-7

Zhu, X., Chen, B., Avadhanam, R. M., Shui, H., \& Zhang, R. Z. (2020). Reading and connecting: Using social annotation in online classes. Information and Learning Sciences, 121(5/6), 261-271. 